\title{
Mejoramiento genético de cebada en el INIFAP (1985-2020)
}

\author{
Miguel González González ${ }^{1 \S}$ \\ Mauro R. Zamora Díaz \\ Salomón Solano Hernández ${ }^{2}$ \\ Ramón Huerta Zurita ${ }^{2}$ \\ René Gómez Mercado ${ }^{3}$ \\ Israel Rojas Martínez ${ }^{4}$
}

${ }^{1}$ Campo Experimental Valle de México-INIFAP. Carretera los Reyes-Texcoco km 13.5, Coatlinchán, Texcoco, Estado de México. (zamora.mauro@inifap.gob.mx). ${ }^{2}$ Campo Experimental Bajío-INIFAP. Carretera Celaya-San Miguel de Allende km 6.5, Celaya, Guanajuato. (solano.salomon@inifap.gob.mx). ${ }^{3}$ Sitio Experimental Hidalgo-INIFAP. Carretera Pachuca-Ciudad Sahagún km 3.6, \# 2000, Torre Norte $1^{\text {er }}$ Piso Despacho 111, Pachuca, Hidalgo. (gomez.rene@inifap.gob.mx). ${ }^{4}$ Sitio Experimental TlaxcalaINIFAP. Carretera Tlaxcala-Chiautempan km 2.5, Chiautempan, Tlaxcala. (rojas.israel@inifap.gob.mx).

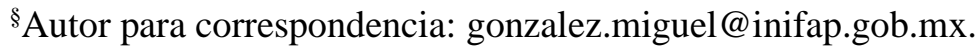

\section{Resumen}

El mejoramiento genético de cebada en México se inició en el año de 1954 en cooperación con la Industria Maltera Nacional, la Secretaría de Agricultura y Ganadería y la Fundación Rockefeller. En el año de 1959 se incrementó la primera variedad mejorada obtenida por el programa de cebada del INIA, Toluca I. Desde sus inicios, el programa de cebada del INIFAP se ha planteado el objetivo de obtener variedades que por su calidad y rendimiento satisfagan tanto las necesidades del agricultor como de los industriales malteros y cerveceros. Por esta razón, las investigaciones de mejoramiento se han enfocado en el incremento del rendimiento, la precocidad y la calidad, además de la resistencia a las enfermedades más comunes del cultivo. Entre 1959 a la fecha, el Programa Nacional de Cebada ha liberado 22 variedades de cebada (17 malteras y 5 forrajeras). Con la creación del INIFAP en 1985, el programa continúo con los trabajos de investigación y obtención de genotipos adecuados para maltería y cervecería. En estos 35 años, se han obtenidos grandes logros en la liberación de variedades, siendo las más importantes Esperanza y Esmeralda que por su resistencia a roya lineal amarilla, permitieron la producción de cebada para cervecería tanto en condiciones de riego como de temporal; logrando la autosuficiencia nacional en el año 2000. Con estas variedades se sembraron más de seis millones de hectáreas en México, más de 300 000 ha anuales y se beneficiaron más de 50000 familias en el país.

Palabras clave: calidad, mejoramiento genético, variedades.

Recibido: febrero de 2021

Aceptado: mayo de 2021 
El desarrollo de cebadas malteras en el país comenzó con el establecimiento de la primera fábrica de malta en la ciudad de México en el año de 1906. La cebada es la materia prima para la elaboración de cerveza. Con el establecimiento de la Oficina de Estudios Especiales (OEE) en 1943, en el Campo Experimental 'El Horno' hoy, Campo Experimental Valle de México, se inician durante 1946 trabajos de mejora en cebada; sin embargo, las cebadas producidas no cumplían con los requerimientos de calidad demandas por los consorcios cerveceros.

Por esta razón, en el año de 1954 se inició el mejoramiento genético de la cebada maltera en México en cooperación con la Industria Maltera Nacional, la Secretaría de Agricultura y Ganadería (SAG) y la Fundación Rockefeller. Los primeros resultados de investigación fueron obtenidos en el año de 1959 con la primera variedad mejorada obtenida por el programa de cebada del Instituto Nacional de Investigaciones Agrícolas (INIA), denominada Toluca I (Zamora et al., 2012).

Entre los años 50's a los 70's, los principales problemas con enfermedades consistían en roya de la hoja, roya del tallo, carbones cubierto y desnudo y enfermedades foliares que afectaban la calidad maltera de la cebada, siendo primordial que las variedades generadas tuvieran calidad maltera, buen rendimiento y tolerancia a estas enfermedades (Riojas, 1973). Desde su creación, el programa de cebada del Instituto Nacional de Investigaciones Forestales, Agrícolas y Pecuarias (INIFAP) se ha planteado el objetivo de obtener variedades que por su calidad y rendimiento satisfagan tanto las necesidades del agricultor como de los industriales malteros y cerveceros tanto para condiciones de riego en el Bajío como para temporal en los Valles Altos del Centro de México.

A la fecha, el programa de cebada ha liberado 22 variedades de cebada (17 malteras y 5 forrajeras), en tres campos generadores: CE Valle de México (CEVAMEX), para áreas de temporal, CE Bajío (CEBAJ), para zonas de riego y CE Valle del Yaqui (CEVY, ahora Campo Experimental Norman E. Borlaug CENEB), en este último, los trabajos de investigación para zonas de riego permitieron obtener cinco variedades destacando Rumorosa y Cucapah 87, variedades forrajeras para el Noroeste del país además de las variedades Centinela y Cerro Prieto que, junto con Puebla (obtenida en el CEBAJ) fueron de vital importancia para los sistemas de producción de riego y temporal en México, así como para la industria maltera, permitiendo autosuficiencia de cebada en el país durante la década de 1980 (Navarro, 1983; Zamora et al., 2012). Actualmente, solo en los campos experimentales del CEVAMEX y CEBAJ se realiza mejoramiento genético, continuando como sede del programa, el CEVAMEX.

Debido a la presencia de la enfermedad denominada roya lineal amarilla (RLA) causado por Puccinia striiformis f. sp, hordei Eriks, los trabajos de investigación se intensificaron pues en 1988, esta enfermedad causó pérdidas de hasta $50 \%$ de los rendimientos de todas las variedades de cebada mejoradas mexicanas, que se sembraban en temporal en los Valles Altos Centrales del país. Pérdidas de menor cuantía se determinaron en las siembras del Bajío en condiciones de riego.

Para controlar esta enfermedad, en 1989 fue liberada la variedad Esperanza para siembras de riego en el Bajío y en 1992, la variedad Esmeralda para condiciones de temporal en los Valles Altos Centrales (González et al., 2006). Actualmente las variedades liberas por el programa de cebada son tolerantes a las principales enfermedades de ambas condiciones de cultivo en México, con excelente rendimiento y con la calidad requerida por la industria maltera-cervecera. 
Las estrategias de investigación aplicadas por el INIA entre 1960 y 1980 en coordinación con la industria maltera-cervecera, fueron determinantes para generar variedades con alto rendimiento y buena calidad maltera, permitiendo incrementar la superficie cultivada con cebada y diversificar la agricultura en varias regiones del país. La obtención de mejores cosechas hizo posible el reducir considerablemente las importaciones; para ello, la participación de Impulsora Agrícola, SA (IASA), fue fundamental. IASA fue creado en 1958 por las industrias cerveceras de la época (Cuauhtémoc, Moctezuma y Modelo) con la encomienda de lograr autosuficiencia del país en la producción de cebada maltera e incrementar el nivel de vida de los productores cebaderos. Sus actividades eran de vital importancia, pues cumplía una función importante en la cadena productiva, promovía la difusión del cultivo, además de que fungía como vínculo entre los industriales cerveceros y el INIA hoy INIFAP, convirtiéndose al mismo tiempo en un importante vehículo del cambio tecnológico de la cebada (IASA, 1983; Aguilar y Schwentesius, 2004).

\section{INIFAP: 35 años de investigación en el cultivo de cebada}

En agosto de 1985, con la fusión del INIA, INIP e INIF, se crea el INIFAP. En esta nueva etapa, el programa de cebada continúo con su objetivo de realizar mejoramiento genético para desarrollar y generar variedades con rendimiento, calidad y tolerante a enfermedades que la industria malteracervecera demandaba. Desde su formación, el Programa Nacional de Cebada del INIFAP, ha participado en el desarrollo de la mayoría de las variedades liberadas en México, tanto para condiciones de riego como para temporal.

Actualmente, las enfermedades son la principal limitante del cultivo de cebada, provocando disminución del rendimiento hasta $100 \%$. La roya lineal amarilla, reportada por primera vez en México en 1987, es considerada el problema más grave. Durante el periodo de 1960 a 1980 el incremento en el rendimiento de grano fue vital en el crecimiento de la producción de cebada del país. Sin embargo, a finales de los 80's estos logros se desvanecieron, por la presencia de RLA.

Para solucionar esta epifitia, la experiencia derivada del manejo del cultivo y del proceso de mejoramiento genético, aunada a la constante evaluación de la calidad maltera y tolerancia a enfermedades, fueron factores decisivos para obtener de manera oportuna variedades tolerantes a RLA. La variedad Esperanza liberada en 1989, fue la primera variedad maltera en México de característica semienana, con potencial de rendimiento de grano superior a $8 \mathrm{t} \mathrm{ha}^{-1}$ en condiciones de riego en El Bajío. Para condiciones de temporal en los Valles Altos de México se liberó en 1992 la variedad Esmeralda, es la primera variedad maltera con tolerancia a RLA (Zamora et al., 2015).

Con el desarrollo de estas variedades se logró salvar la producción de cebada maltera en las dos condiciones de producción: riego y temporal, logrando la autosuficiencia nacional en el año 2000, generando beneficios importantes para productores e industriales. Con estas variedades se sembraron más de 6 millones de hectáreas en México, más de 300000 ha anuales y se beneficiaron más de 50000 familias (UPR) en el país. Los beneficios ambientales, aunque no cuantificables, son enormes, pues la buena resistencia de estas variedades a RLA evitó la aplicación de fungicidas en ambas condiciones de producción (González et al., 2006).

A inicios del presente siglo, los trabajos de investigación desarrollados por el Programa Nacional de Cebada permitieron la obtención de las variedades Adabella en 2004, Armida en 2005 y Alina en 2006 (Zamora et al., 2015). La primera recomendada para condiciones de temporal, mientras 
que las otras dos, recomendadas para condiciones de riego en el Bajío. Estas variedades poseen mejores características, buena calidad industrial y con mayor rendimiento que sus antecesores. En 2016, fue liberado comercialmente la variedad Maravilla, cebada con atributos forrajeros (Zamora et al., 2017).

Los avances en calidad maltera han sido continuos desde el inicio de la investigación de este cultivo en México. En materiales de seis hileras se ha incrementado anualmente $0.07 \%$ el extracto, mientras que el contenido de proteína se ha reducido $0.04 \%$ por año. De acuerdo con Schwarz y Horsley (1995) en el transcurso de 1910 a 1990, los programas de mejoramiento genético de cebada maltera en Estados Unidos de América, incrementaron 0.06\% el extracto y redujeron $0.01 \%$ el contenido de proteína anualmente.

El laboratorio de calidad de cebada interactúa estrechamente con el área de mejoramiento genético del Programa Nacional de Cebada, evaluando la calidad de las líneas segregantes $\left(\mathrm{F}_{4}-\mathrm{F}_{7}\right)$ hasta generaciones avanzadas $\left(>\mathrm{F}_{7}\right)$ obtenidas durante el proceso de selección. En generaciones segregantes se evalúan aspectos de calidad relacionados con el contenido de proteína y tamaño del grano; mientras que en generaciones avanzadas se analizan características inherentes del grano para el proceso de malteo y calidad de malta, basándose en los parámetros establecidos en la norma mexicana NMX-FF-043-SCFI-2003 (calidad de cebada) y por la Industria Maltera nacional (calidad de malta) (Zamora et al., 2017).

La fortaleza del mejoramiento genético del programa de cebada radica en que las investigaciones realizadas responden a la demanda de los industriales maltero-ceverceros, quienes mediante convenios financiaron las investigaciones que permitieron liberar variedades malteras. Los proyectos financiados con recursos del INIFAP, han contribuido en la diversificación de este importante cereal en otros sectores de la cadena agroalimentaria del país. En estos 35 años de mejoramiento genético, se han mantenido las metodologías convencionales (método de mejoramiento por Pedigree o genealógico) y se han incorporado otras estrategias (combinaciones en entre métodos de mejoramiento: Pedigree, masivo, individual; además, de pruebas in situ y ex situ para tolerancia a enfermedades, entre otros) que permiten optimizar los recursos para obtener genotipos con características adecuadas a diferentes sistemas y sectores de producción en el país.

Las actividades prioritarias de investigación en cebada están relacionadas con el rendimiento y la calidad. Para ello, se evalúa la interacción genotipo-ambiente, se determinan las pérdidas ocasionadas por royas y enfermedades foliares, su identificación, distribución y análisis de la resistencia a las royas, resistencia a plagas, uso eficiente del agua. Además, se realizan análisis de calidad industrial, permitiendo la selección de genotipos con la calidad requerida.

Los trabajos de mejoramiento genético realizadas de manera alternada en los campos experimentales Valle de México y Bajío, permiten acortar a la mitad el tiempo de selección y obtención de líneas avanzadas, los cuales son evaluados en ambientes contrastantes. La evaluación involucra zonas estratégicas de diferentes regiones productoras de cebada bajo condiciones de temporal, de los estados de Hidalgo, Tlaxcala, Puebla, Estado de México, Guanajuato, Sonora, Durango, Coahuila, Aguascalientes, Tamaulipas, Chihuahua, Jalisco, Zacatecas y Oaxaca, en donde se seleccionan genotipos con potencial de rendimiento, tolerantes a enfermedades y estables en la mayoría de los ambientes (Zamora et al., 2015; González et al., 2016). 
En el programa nacional de cebada durante estas dos etapas (INIA e INIFAP), a través de los trabajos de investigación realizados mediante el convenio tripartita Industria Cervecera-IASAINIFAP, han colaborado diversos investigadores de al menos 16 campos y sitios experimentales distribuidos en seis regiones a lo largo y ancho del país, cuya participación ha sido de vital importancia en los diferentes proyectos que aborda el programa: mejoramiento genético, laboratorio de calidad, manejo integrado de enfermedades, productividad de agrosistemas, manejo integrado de malezas, manejo integrado de plagas, uso y manejo del agua y transferencia de tecnología (Zamora et al., 2009).

\section{Retos a corto, mediano y largo plazo}

Desde su creación, el programa de cebada del INIFAP, ha generado variedades adecuadas para la industria maltera-cervecera. Actualmente, la demanda hacia este sector requiere de variedades de dos hileras, con potencial de rendimiento, calidad (bajo contenido de proteína y $\beta$-glucanos y alto contenido de almidón) y tolerantes a enfermedades, mientras que, en otros sectores, como el pecuario, las investigaciones se centran en la generación de genotipos de seis hileras y sin aristas, con potencial forrajero (calidad y rendimiento, entre otras variables de interés).

Las recientes variaciones ambientales, hacen necesaria la obtención de nuevos cultivares con calidad maltera adaptados a una amplia gama de condiciones de estrés biótico y abiótico (Zamora et al., 2015). Las enfermedades en el cultivo de cebada son la principal limitante y afectan la calidad y rendimiento del grano por lo que la resistencia de las variedades a las enfermedades es indispensable. El INIFAP es pionero y referente en la generación de variedades de cebada para la industria cervecera, la experiencia obtenida en este sector facilita aplicar los conocimientos de investigación en otras áreas permitiéndole al instituto continuar a la vanguardia en la generación de variedades de cebada para beneficio de los productores de México.

\section{Conclusiones}

El programa nacional de cebada del INIFAP, ha liberado 17 variedades de cebada maltera y cinco de cebada forrajera. Las variedades más importantes son Esperanza y Esmeralda que por su resistencia a roya lineal amarilla, permitieron la producción de cebada para cervecería tanto en condiciones de riego como de temporal, logrando la autosuficiencia nacional en el año 2000. Las investigaciones agronómicas en las principales áreas productoras de este cereal se enfocan a resolver la problemática sobre fechas y densidades de siembra, fertilización, control de malezas y plagas y uso y manejo del agua, los resultados han contribuido a incrementar el rendimiento y la calidad industrial del grano de forma significativa.

\section{Literatura citada}

Aguilar, A. J. y Schwentesius, R. R. 2004. La producción de la cebada maltera en México: ventaja comparativa no capitalizada. Reporte de Investigación 72. Primera (Ed.). Centro de Investigaciones Económicas, Sociales y Tecnológicas de la Agroindustria y la Agricultura Mundial (CIESTAAM). Universidad Autónoma Chapingo. Estado de México. 62 p. 
González, E. A.; Zamora, D. M.; Márquez, C. L. A.; Ramírez, P. F.; Ibañez, A. M.; Islas, G. J. y Wood, S. 2006. Impacto económico del mejoramiento genético de la cebada en México: variedad Esmeralda. Serie: estudios de evaluación del impacto económico de productos del INIFAP. Publicación técnica núm. 19.69 p.

González, G. M.; Zamora, D. M. y Solano, H. S. 2016. Evaluación agronómica y física en líneas avanzadas de cebada maltera. Rev. Mex. Cienc. Agríc. 7(1):159-171.

IASA. 1983. Impulsora Agrícola S. A. El cultivo del a cebada maltera de temporal. Impulsora Agrícola, SA. México, DF. Folleto informativo. 68 p.

Navarro, F. M. 1983. Logros y aportaciones de la investigación agrícola en el cultivo de la cebada. Publicación especial Núm. 107. SARH, INIA. México, DF. 5-16 pp.

Riojas, G. E. 1973. Variedades mexicanas de cebada. INIA, SAG. México. Folleto de divulgación núm. 49.20 p.

Schwarz, P. B. and Horsley, D. 1995. Malt quality improvement in North American six-rowed barley cultivars since 1910. J. Am. Soc. Brewing Chem. 53(1):14-18.

Zamora, D. M. R.; Pérez, R. J. A.; Huerta, Z. R.; López, C. M. L.; Gómez, M. R. y Rojas, M. I. 2017. Maravilla: variedad de cebada forrajera para Valles Altos de México. Rev. Mex. Cienc. Agríc. 8(6):1449-1454.

Zamora, D. M.; Solano, H. S.; Huerta, Z. R. 2009. L a cebada maltera (Hordeum vulgare L.) cereal fundamental en la historia del Campo Experimental Valle de México. In: Reseña histórica 66 años de investigación al servicio de México 1943-2009. Publicación especial núm. 1. 45-49 pp.

Zamora, D. M.; Solano, H. S.; Huerta, Z. R.; González, G. M. y López, C. M. L. 2012. Programa Nacional de Cebada del INIFAP: cinco décadas de investigación aplicada. In: Día de Campo CEVAMEX 2012. Memoria técnica núm. 13. 137-140 pp.

Zamora, D. M.; Solano, H. S.; Pérez, R. J. A.; López, C. M. L.; Gómez, M. R.; Rojas, M. I. y Huerta, Z. R. 2015. Avances y perspectivas del cultivo de cebada en México. In: contribuciones científicas y tecnológicas del Campo Experimental Valle de México a 30 años de la creación del INIFAP y retos ante el cambio climático. Memoria técnica núm. 2. 116-127 pp. 